ERRATA-CORRIGE

\title{
FINITELY ADDITIVE CONDITIONAL PROBABILITIES
}

\author{
by \\ EUGENIO REGAZZINI
}

Rendiconti del Seminario Matematico e Fisico di Milano (1985) 55, 69-89.

Patrizia Berti has kindly pointed out to me a gap in the proof of Theorem 7 (pp. 84-85). In fact, $T_{2}$-measurability of $f$ w.r.t. $(\Omega, \mathcal{F})$ need not follow from $T_{2}$-measurability of $f$ w.r.t. $(\Omega, \mathscr{H})$. Hence, the first period of the proof is incorrect. The problem can be overcome by assuming that $f$ is $T_{2}$-measurable w.r.t. $(\Omega, \mathcal{F})$. 